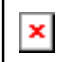

\title{
The Biased Transformation and its Application in Goodness- of-Fit Tests for the Beta and Gamma Distribution
}

\begin{tabular}{|r|l|}
\hline Journal: & Communications in Statistics - Simulation and Computation \\
\hline Manuscript ID: & LSSP-2009-0039.R1 \\
\hline Manuscript Type: & Original Paper \\
\hline Date Submitted by the \\
Author: & 22-Jun-2009 \\
\hline Complete List of Authors: & Raschke, Mathias; Fa. M. Raschke \\
\hline Keywords: & $\begin{array}{l}\text { beta distribution, gamma distribution, goodness-of-fit test, } \\
\text { transformation }\end{array}$ \\
\hline Abstract: & $\begin{array}{l}\text { The biased transformation is introduced for continuous random } \\
\text { variables. It is proved in the paper that the biased transformation } \\
\text { works for the generalized Pareto, the generalized extreme value } \\
\text { and the normal distribution. The biased transformation works for } \\
\text { continuous distributions of the exponential family as an } \\
\text { approximation; this is demonstrated with the aid of simulations. } \\
\text { The new transformation can be used for the Anderson-Darling test } \\
\text { for the gamma and beta distribution. The power of the new test } \\
\text { procedure is validated by simulations. The practical relevance of the } \\
\text { new test for the beta distribution is demonstrated in an analysis of } \\
\text { meteorological data. }\end{array}$ \\
\hline
\end{tabular}

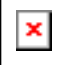


Biased transformation and Goodness-of-Fit Test

\title{
The Biased Transformation and its Application in Goodness-of-Fit Tests for the Beta and Gamma Distribution
}

\author{
Mathias Raschke \\ Fa. M. Raschke \\ Teichgasse 18, 99423 Weimar, Germany \\ Phone $+49 \mid(0) 36431903238$ \\ E-Mail MathiasRaschke@t-online.de
}

\begin{abstract}
The biased transformation is introduced for continuous random variables. It is proved in the paper that the biased transformation works for the generalized Pareto, the generalized extreme value and the normal distribution. The biased transformation works for important continuous distributions of the exponential family as an approximation; this is demonstrated with the aid of simulations. The new transformation can be used for the Anderson-Darling test for the gamma and beta distribution. The power of the new test procedure is validated by simulations. Finally, a first powerful test is available for the beta distribution although the power function is poor. The $\chi^{2}$-test has lower power. The Kolomogorov-Smirnov test for a fully specified distribution has almost no power in the application for the beta distribution with estimated parameters. The practical relevance of the new test for he beta distribution is demonstrated in an analysis of meteorological data.
\end{abstract}

Keywords: beta distribution, gamma distribution, goodness-of-fit test, transformation

\section{Introduction}

The following transformation and test procedures have been researched by chance as the author was trying out different transformation methods in flood statistics and was looking into publications for a goodness-of-fit test for the gamma distribution. It is possible to use the found biased transformation to test the goodness-of-fit for the gamma distribution. But there exist already efficient tests for the gamma distribution (Stephens 1986). Except this, there is no practicable and specific goodness-of-fit test for the beta distribution so far. This was the motivation to research the biased transformation for some random distribution and to research the power of the test using biased transformation for the gamma and the beta distribution.

The beta and the gamma distributions are very famous and are explained in different publications e.g. by Johnson et al. (1994, 1995). 
Biased transformation and Goodness-of-Fit Test

The goodness-of-fit of an estimated distribution can be tested by the Chi-Squared test for a sample. This and related tests are explained by Moore (1986). There is no special consideration of the type of distribution in this test. The EDF-tests (empirical distribution function tests) consider the type of distribution. Different types of EDF-tests are published like the Anderson Darling test (Anderson and Darling 1952, 1954), Cramer von Mieses Test and Watson Test both introduced by Watson (1961), the Kuiper test (Kuiper 1960) and the famous KolmogorovSmirnov test (Kolmogorov 1933). The critical values of EDF-test are published by Stephens in different publications $(1974,1976)$ for different types of distributions. A summary for the EDFtest for different types of distributions is given by Stephens (1986). The EDF-test for normality with the best power is the Anderson Darling test (Landry and Lepage 1992). The moment tests are not often used. The basics of this test type are explained by Bowman and Shento (1986). The theory of a moment test is discussed for beta distributions by Li and Papadopoulos (2002) but contains a mistake (a moment is divided by itself) and the power of this test approach is small (Li and Papadopoulos 2002, Tab. 1-3).

Generally each continuously variable can be transformed into another random variable by any continuously function which is defined in the ranges of these random variables. The power transformation of Box and Cox (1964) is an important family of transformation. The goal of these transformations is the normalisation of the random variable. A further transformation implies the application of Copulas (Sklar 1959, Carley and Taylor 2000). The continuously random variables are transformed to uniform distributed variables by using the marginal distribution or by using the EDF. The approach to use a transformation for goodness-of-fit test is introduced already by Quesenberry (1986).

The biased transformation (BT) is introduced here and is explained in the second section. In the third section is shown that BT works as an approximation for the normal, gamma and beta distribution. Furthermore the BT is applied in an EDF- test procedure for the gamma and the beta distribution in sections 4 and 5 . The benefit of the new test procedure for the beta distribution is demonstrated in the section 6 with weather data. The results are summarised and discussed in the last section. The theoretical research for the BT for the generalised Pareto 
Biased transformation and Goodness-of-Fit Test

distribution (GPD), the generalised extreme value distribution (GEV) and the normal distribution is given in the appendix.

\section{The Biased Transformation}

A continuous random variable $X_{A}$ with cumulated distribution function $(C D F) F_{A}$ can generally be transformed to another $\mathrm{X}_{\mathrm{B}}$ with $\mathrm{CDF} \mathrm{F}_{\mathrm{B}}$ with the transformation function $\mathrm{x}_{\mathrm{B}}\left(\mathrm{x}_{\mathrm{A}}\right)$, which is

$$
x_{B}=F_{B}^{-1}\left[F_{A}\left(x_{A}\right)\right] .
$$

$\mathrm{F}^{-1}$ is the symbol for the inverse function with $\mathrm{x}=\mathrm{F}^{-1}(\mathrm{~F}(\mathrm{x}))$. A further biased transformation (BT) is introduced here with

$$
x_{D}=F_{C}^{-1}\left[F_{B}\left(x_{A}\right)\right] .
$$

The type of $C D F F_{A}$ and $F_{B}$ is identical and likewise the type of $C D F F_{C}$ and $F_{D}$ is identical, but the parameter vectors $\theta$ don't need to be equal $-\theta_{A} \neq \theta_{B}$ and $\theta_{C} \neq \theta_{D}$. Furthermore the type of $F_{A}$ and $F_{B}$ need not to be identical to distribution $\mathrm{F}_{\mathrm{C}}$ and $\mathrm{F}_{\mathrm{D}}$. The functions and distributions are distinguished by the capitals A to D. All CDF are continuous in the relevant ranges. The probability density function (PDF) is the derivative $\mathrm{f}(\mathrm{x})$ of the $\mathrm{CDF} F(\mathrm{x})$ with respect to the random variable. This includes the assumption that the $\mathrm{CDF}$ is differentiable in the relevant range. The $C D F \mathrm{~F}_{\mathrm{B}}$ and $\mathrm{F}_{\mathrm{C}}$ are of interest and be used in this paper. The corresponding random variables $X_{B}$ and $X_{C}$ are not used here.

The transformation implies that

$$
\begin{gathered}
F_{D}\left[x_{D}\left(x_{A}\right)\right]=F_{A}\left(x_{A}\right) \text { and } \\
f_{D}\left[x_{D}\left(x_{A}\right)\right]=f_{A}\left(x_{A}\right) f_{C}\left[x_{D}\left(x_{A}\right)\right] / f_{B}\left(x_{A}\right) .
\end{gathered}
$$

The transformation is called "biased" because $\mathrm{F}_{\mathrm{A}}\left(\mathrm{x}_{\mathrm{A}}\right) \neq \mathrm{F}_{\mathrm{B}}\left(\mathrm{x}_{\mathrm{A}}\right)$ in equation 2 . The biases are described by the bias function $\mathrm{F}_{\mathrm{B}}\left(\mathrm{F}_{\mathrm{A}}\right)$ where $\mathrm{F}_{\mathrm{B}}\left(\mathrm{F}_{\mathrm{A}}\right)=\mathrm{F}_{\mathrm{B}}\left(\mathrm{x}_{\mathrm{A}}\right)$ with $\mathrm{F}_{\mathrm{A}}\left(\mathrm{x}_{\mathrm{A}}\right)$. Equation 2 is the transformation $\mathrm{x}_{\mathrm{D}}\left(\mathrm{x}_{\mathrm{A}}, \theta_{\mathrm{B}}, \theta_{\mathrm{C}}\right)$, and from this the functions $\theta_{\mathrm{D}}\left(\theta_{\mathrm{A}}, \theta_{\mathrm{B}}, \theta_{\mathrm{C}}\right)$ can be deduced. The transformation is an assumption until it is proved for the concrete distribution types. It has to be shown in a proof that either equation 3 or 4 is right for the functions $\mathrm{x}_{4}\left(\mathrm{x}_{1}, \theta_{2}, \theta_{3}\right)$ and $\theta_{4}\left(\theta_{1}, \theta_{2}, \theta_{3}\right)$.

The BT works for some distributions. This is demonstrated by the research of the generalised Pareto distribution (GPD) and the generalised extreme value distribution (GEV). The distributions, 
Biased transformation and Goodness-of-Fit Test

theorems and simple proofs are formulated in the appendix. Furthermore, the BT works exactly for the normal distribution with the PDF

$$
f(x)=1 /(\sigma \sqrt{2 \pi}) \exp \left[-(x-\mu)^{2} /\left(2 \sigma^{2}\right)\right], \quad \sigma>0
$$

The theorem and the proof for BT of the normal distributed $X_{A}$ and $X_{D}$ is described in the appendix.

\section{The BT as an Approximation for Some Continuous Distributions of the Exponential Family}

The exponential family includes the normal, the gamma and the beta distribution. The PDF for the gamma distribution is denoted $\Gamma(\alpha)$ as a gamma function of $\alpha$ with

$$
f(x)=(x / \lambda)^{\alpha-1} \exp (-x / \lambda) /[\lambda \Gamma(\alpha)], \quad x \geq 0, \lambda>0, \alpha>0 .
$$

The PDF for the beta distribution is denoted $\mathrm{B}(\alpha, \beta)$ as beta function of $\alpha$ and $\beta$ with

$$
f(x)=x^{\alpha-1}(1-x)^{\beta-1} / \mathrm{B}(\alpha, \beta), \quad 0 \leq x \leq 1, \alpha>0, \beta>0 .
$$

There are no elementary solutions for the integral of the CDF of the beta distribution, the gamma distribution and the normal distribution. But the CDF can be computed numerically.

The BT can be used for the gamma, beta and normal distribution as an approximation.

Assumption: If $\mathrm{X}_{\mathrm{A}}$ with $\mathrm{F}_{\mathrm{A}}$ is normal, beta or gamma distributed, and $\mathrm{F}_{\mathrm{B}}$ is the same type of $\mathrm{CDF}$ as $\mathrm{F}_{\mathrm{A}}$ with $\theta_{A} \neq \theta_{B}$ but not with $\left|\theta_{A}-\theta_{B}\right|>>0$, then $X_{A}$ can be transformed with $F_{C}$ according equation 2 to $X_{D}$. $F_{C}$ has any $\theta_{c}$ and $F_{D}$ of $X_{D}$ can be approximated by the same type of $F_{C}$.

The basic idea for the validation is the following. Samples of a uniform distributed random variable $U$ $(0 \leq \mathrm{u} \leq 1)$ can be simulated. From this sample, the sample of $\mathrm{X}_{\mathrm{A}}$ can be computed by using the inverse function with defined and simulated values of $\theta_{\mathrm{A}}$. The sample of $X_{D}$ can be computed with the sample of $\mathrm{X}_{\mathrm{A}}$ and any defined and simulated values of $\theta_{\mathrm{B}}$ and $\theta_{\mathrm{C}}$. The parameter vector of the samples of $\mathrm{X}_{\mathrm{A}}$ and $X_{D}$ can be estimated by the maximum likelihood method (ML). The goodness-of-fit of $F_{A}\left(x_{A}, \hat{\theta}_{A}\right)$ and $\mathrm{F}_{\mathrm{D}}\left(\mathrm{x}_{\mathrm{D}}, \hat{\theta}_{\mathrm{D}}\right)$ can be evaluated with the real probability which is the simulated and known sample of U. The validation values of goodness-of-fit are defined here with $\Delta_{\max }$ and $\Delta^{2}$

$$
\Delta_{\max }=\max _{i=1 . . n}\left|u_{i}-F\left(x_{i}, \hat{\theta}\right)\right|
$$


Biased transformation and Goodness-of-Fit Test

$$
\Delta^{2}=\frac{\sum_{i=1}^{n}\left[u_{j}-F\left(x_{i}, \hat{\theta}\right)\right]^{2}}{n} .
$$

The definition of $\Delta_{\max }$ is comparable with the test value of the Kolmogorov-Smirnov test of fit. The definition of $\Delta^{2}$ is similar to the mean squared error of parameter estimation. The values $\Delta_{\max }$ and $\Delta^{2}$ are random variables as well. If the assumption is right for the defined range of parameters of transformation $\theta_{\mathrm{B}}$ and $\theta_{\mathrm{C}}$, then the distribution of $\Delta_{\max }$ and $\Delta^{2}$ of $\mathrm{X}_{\mathrm{A}}$ is equal or very similar to the distribution of $\Delta_{\max }$ and $\Delta^{2}$ of $\mathrm{X}_{\mathrm{D}}$; and $\Delta_{\max }$ and $\Delta^{2}$ should be strongly correlated for $\mathrm{X}_{\mathrm{A}}$ and $\mathrm{X}_{\mathrm{D}}$.

Simulations have been carried out in a broad range of parameters and sample sizes to validate the assumption. $\theta_{A}$ and $\theta_{C}$ are simulated in a defined range. $\theta_{B}$ have to be defined with $\theta_{B}=\theta_{A}+\Delta_{\theta}$ with the simulated $\theta_{\mathrm{A}}$ and a simulated $\Delta_{\theta}$ in a defined range of $\Delta_{\theta}$ which is rather narrow. If the difference $\Delta_{\theta}$ is to large the approximations of $\mathrm{F}(\mathrm{x})$ for the normal, beta or gamma distribution could result in $\mathrm{F}_{\mathrm{B}}\left(\mathrm{x}_{\mathrm{A}}\right)=1$ or $\mathrm{F}_{\mathrm{B}}\left(\mathrm{x}_{\mathrm{A}}\right)=0$. If $\mathrm{F}_{\mathrm{C}}^{-1}(1)$ or $\mathrm{F}_{\mathrm{C}}^{-1}(0)$ results in infinity or negative infinity, the transformation has to be cancelled.

The empirical distributions of $\Delta_{\max }$ and $\Delta^{2}$ are plotted here for graphical comparisons with the position

$$
\hat{P}_{i}=i /(n+1)
$$

The Kolomogorov-Smirnov test for homogeneity (Birnbaum and Hall 1960) is used to validate that the distributions of $\Delta_{\max }$ of $\mathrm{X}_{\mathrm{A}}$ and $\Delta_{\max }$ of $\mathrm{X}_{\mathrm{D}}$ are the same population and the distributions of $\Delta^{2}$ of $\mathrm{X}_{\mathrm{A}}$ and $\Delta^{2}$ of $X_{D}$ are the same population. The test value $D_{n 1, n 2}$ of the test of homogeneity of the two the samples of $\Delta^{2}$ is written

$$
D_{n 1, n 2}=\max _{\Delta^{2}}\left\{\left|\hat{F}_{1}\left(\Delta^{2}\right)-\hat{F}_{2}\left(\Delta^{2}\right)\right|\right\}
$$

and for $\Delta_{\max }$ in the same manner. The test values should be smaller than the critical value for a significance of $\alpha=10 \%$ or higher. $H_{0}$ (the same population) is not rejected.

Three variants for the sample size $\mathrm{n}$ for $\mathrm{X}_{\mathrm{A}}$ and $\mathrm{X}_{\mathrm{D}}$ were simulated with $\mathrm{n}=10,100$ and 1000. For each variant of sample size and distribution type of $F_{A}$ and $F_{D}, 1000$ simulations were carried out. The ML estimation of the parameters was carried out for beta and gamma distributions with an exactness of $10^{-4} \%$. For the different variants, the following ranges of parameters were defined: 
Biased transformation and Goodness-of-Fit Test

- If $X_{A}$ is gamma distributed, then the parameters were simulated with $\alpha_{A}=1.0 \ldots 3.5$ and $\lambda_{A}=0.5 \ldots 4.0$ uniformly distributed. The parameters of $F_{B}$ were simulated with $\alpha_{B}=\alpha_{A}+\Delta_{\alpha}, \Delta_{\alpha}=-$ $0.2 \ldots 0.2$ uniformly distributed and $\lambda_{B}=\lambda_{A}+\Delta_{\lambda}, \Delta_{\lambda}=-0.2 \ldots 0.2$ uniformly distributed.

- If $X_{A}$ is beta distributed the parameters were simulated with $\alpha_{A}=0.75 \ldots 4.0$ and $\beta_{A}=0.75 \ldots 4.0$ uniformly distributed. The parameters of $F_{B}$ were simulated with $\alpha_{2}=\alpha_{A}+\Delta_{\alpha}, \Delta_{\alpha}=-0.25 \ldots 0.25$ uniformly distributed and $\beta_{\mathrm{B}}=\beta_{\mathrm{A}}+\Delta_{\beta}, \Delta_{\beta}=-0.25 \ldots 0.25$ uniformly distributed.

- If $X_{A}$ is normally distributed, the parameters were simulated with $\mu_{A}=-0.5 \ldots 0.5$ and $\sigma_{A}=0.75 \ldots 1.25$ uniformly distributed. The parameters of $F_{B}$ were simulated with $\mu_{B}=\mu_{A}+\Delta_{\mu}$, $\Delta_{\mu}=-0.25 \ldots 0.25$ uniformly distributed and $\sigma_{\mathrm{B}}=\sigma_{\mathrm{A}}+\Delta_{\sigma}, \Delta_{\sigma}=-0.25 \ldots 0.25$ uniformly distributed.

The parameters of $F_{C}$ were simulated independent of $\theta_{A}$ in the following ranges:

- If $\mathrm{F}_{\mathrm{C}}(\mathrm{x})$ is the CDF of a gamma distribution, the parameter $\alpha_{\mathrm{C}}$ was simulated in the range $\alpha_{C}=0.7 \ldots 4.0$ uniformly distributed and the parameter $\lambda_{C}$ was simulated in the range $\lambda_{\mathrm{C}}=0.7 \ldots 4.0$ uniformly distributed.

- If $\mathrm{F}_{\mathrm{C}}(\mathrm{x})$ is the $\mathrm{CDF}$ of a beta distribution, the parameter $\alpha_{\mathrm{C}}$ was simulated in the range $\alpha_{C}=0.7 \ldots 4.0$ uniformly distributed and the parameter $\beta_{C}$ was simulated in the range $\beta_{C}=0.7 \ldots 4.0$ uniformly distributed.

- If $F_{C}(x)$ is the $C D F$ of a normal distribution, the parameter $\mu_{C}$ was simulated in the range $\alpha_{C}=-$ $1 \ldots 1$ uniformly distributed and the parameter $\sigma_{C}$ was simulated in the range $\sigma_{C}=0.5 \ldots 1$ uniformly distributed.

The important point of the BT is the bias function $\mathrm{F}_{\mathrm{B}}\left(\mathrm{F}_{\mathrm{A}}\right)$. The dimension of the bias functions of the simulations are shown by the extreme of $\mathrm{F}_{\mathrm{B}}\left(\mathrm{x}_{\mathrm{A}}\right)$ for the simulated parameters $\theta_{\mathrm{A}}$ and $\theta_{\mathrm{B}}$ and the mean values of the absolute difference $\left|\mathrm{F}_{\mathrm{B}}\left(\mathrm{x}_{\mathrm{A}}\right)-\mathrm{F}_{\mathrm{A}}\left(\mathrm{x}_{\mathrm{A}}\right)\right|$ for the simulated parameters $\theta_{\mathrm{A}}$ and $\theta_{\mathrm{B}}$ as function of $\mathrm{F}_{\mathrm{A}}$ in Fig. 1. The bias of BT of simulated samples is large. The good results of the simulation can not be explained with a small bias in BT. The result of the simulation is a validation of the assumption. It can be seen in Figures $2 \mathrm{a}$ and $\mathrm{b}$ with the distributions of $\Delta_{\max }$ and $\Delta^{2}$ for the case that $\mathrm{X}_{\mathrm{A}}$ is normally distributed. The plotted values of $\Delta_{\max }$ and $\Delta^{2}$ of the different variants of $\mathrm{F}_{\mathrm{C}}$ and $\mathrm{X}_{\mathrm{D}}$ result in very similar curves. It is the same for the case that $\mathrm{X}_{\mathrm{A}}$ is gamma or beta distributed. The test values of the 
Biased transformation and Goodness-of-Fit Test

Kolmogorov-Smirnov test of homogeneity for two samples are in Tab. 1. All values $D_{n 1 ; n 2}$ are much smaller than the critical value of 0.048 for the level of significance of $10 \%$ for the 1000 simulations (number of simulations = sample size here) $\mathrm{H}_{0}-$ the same population - is never rejected. The BT works for the normal, beta and gamma distribution as a good approximation in the range of the simulations. The sample size $\mathrm{n}$ does not influence the result. The correlation of the validation values of goodness-of-fit is very high and in a small range around r=0.98. It is clear in Figures $2 \mathrm{a}$ and $\mathrm{b}$ that the transformation for $\mathrm{X}_{\mathrm{A}}$ to $\mathrm{X}_{\mathrm{D}}$ as normal distribution is exactly as shown in proof in the appendix.

If a beta or gamma distribution is transformed into a normal distribution with transformation parameters of $F_{B}$ which differ not much from the parameters $F_{A}$ of $X_{A}$, than the fit of an estimated normal distribution $F_{D}$ for $X_{D}$ should not be influenced by the parameters of $F_{B}$ and $F_{C}$ according to the simulation results. The parameters of $F_{c}$ should not influence the fit of $X_{D}$ according to the simulation results.

\section{The Application of the BT for the Anderson-Darling Test for the Gamma Distribution}

There are EDF tests of goodness-of-fit with the hypothesis $\mathrm{H}_{0}$ that the sample is from the assumed distribution with the estimated parameters and with the defined level of significance. The level of significance is the share of rejections although the assumption $\mathrm{H}_{0}$ is correct. The transformed variable $\mathrm{X}_{\mathrm{D}}$ has almost the equal quality of goodness-of-fit as the variable $\mathrm{X}_{\mathrm{A}}$ as shown earlier. The results of the goodness-of-fit test should almost be the same for $X_{D}$ and likewise for $X_{A}$. The different shares of rejection of the test hypothesis $\mathrm{H}_{0}$, even if $\mathrm{H}_{0}$ is right - an error of the first kind - should be almost equal. The power should be almost equal likewise. The power is the share of rejection if $\mathrm{H}_{0}$ is false. The error of second kind is the share of acceptance of a false hypothesis $\mathrm{H}_{0}$.

The power function is the probability of rejection dependent on estimated parameter. The parameters of $\mathrm{CDF}$ are the independent variables in the power function. The probability of rejection should increase strong if the absolute difference between the parameters in the power function and the estimated parameters increase. This power function is not good in the test with the BT because the basics of the BT. But the test with the BT may be used only for the ML-estimated parameters. Furthermore, the power of the test can be very high independent of the poor power function. It is 
Biased transformation and Goodness-of-Fit Test

tested with BT if the sample is from a family of distributions. This approach is not new. It has been already introduced by Quesenberry (1986, equation 6.2).

The approach is used concretely for the Anderson-Darling test (Anderson and Darling 1952, Stephens 1974). The test value is $A^{2}$ with

$$
A^{2}=-n-1 / n \sum_{j=1}^{n}\left\{(2 i-1)\left[\ln \left(F\left(x_{i}, \hat{\theta}\right)\right)+\ln \left(1-F\left(x_{n+1-i}, \hat{\theta}\right)\right]\right\} .\right.
$$

The test value has to be adopted for normal distributions with

$$
A^{* 2}=A^{2}\left(1+0.75 / n+2.25 / n^{2}\right) .
$$

The critical values are 1.035 for the level of significance of $1 \%, 0.752$ for the level of significance of $5 \%$ and 0.631 for the level of significance of $10 \%$. Only the Anderson-Darling test is used from the EDF-tests because the Anderson-Darling Test has the best behaviour of the tests for normality according Landry and Leparge (1992). But the approach probably works for the Kolmogorov-Smirnov test and the Cramér-von-Mises test for the goodness-of-fit likewise.

The procedure for the test of goodness-of-fit for the gamma distributed $\mathrm{X}_{\mathrm{A}}$ by using the BT is the following:

- $\quad$ ML estimation of $\hat{\theta}_{\mathrm{A}}$.

- Using this estimation of $\hat{\theta}_{\mathrm{A}}$ for $\theta_{\mathrm{B}}$.

- Definition of $\theta_{C}$ with $F_{C}$ as the CDF of a normal distribution with $\mu=0$ and $\sigma=1$.

- $\quad$ Computing the sample of $\mathrm{X}_{\mathrm{D}}$ by the BT according equation 2 .

- $\quad$ ML estimation of $\hat{\theta}_{\mathrm{D}}$ of the sample of $\mathrm{X}_{\mathrm{D}}$, assumed as normal distribution $-\mathrm{H}_{0}$.

- $\quad$ Computing of the $\mathrm{A}^{2}$ for the sample of $\mathrm{X}_{\mathrm{D}}$ and test.

- Rejection of $\mathrm{H}_{0}$ at the defined level of significance if $\mathrm{A}^{* 2}>\mathrm{A}_{\text {critical }}^{2}$, otherwise acceptance of $\mathrm{H}_{0}$. The assumed gamma distribution of $\mathrm{X}_{\mathrm{A}}$ is rejected with the rejection of the assumed normal distribution of $\mathrm{X}_{\mathrm{D}}$.

The results of the test of the samples of $X_{D}$ from simulated samples of $X_{A}$ can be compared with results of the Anderson-Darling test for estimation of the gamma distribution of $\mathrm{X}_{\mathrm{A}}$ according to Stephens (1986, critical values form Tab. 4.21). Stephens' test procedure is applied for the case that all parameters are estimated except the location parameter. There is no location parameter in the gamma 
Biased transformation and Goodness-of-Fit Test

distribution as formulated above, the location parameter is known and has the value 0 in this paper. Interpolation functions (from regression analysis of values given by Stephens, 1986, Tab. 4.21) are used here for the given critical value for the significance levels of $1 \%, 5 \%$ and $10 \%$ with

$$
\begin{gathered}
A_{\text {critical } 1 \%}^{2}=1.035+0.057 \hat{\alpha}^{-1.093}, \\
A_{\text {critical } 5 \%}^{2}=0.752+0.034 \hat{\alpha}^{-1.114}, \\
A_{\text {critical } 10 \%}^{2}=0.631+0.0264 \hat{\alpha}^{-1.110} .
\end{gathered}
$$

The parameter vector $\theta_{\mathrm{A}}$ for $\mathrm{X}_{\mathrm{A}}$ - assumed to be gamma distributed has to be estimated by the MLmethod. This is defined in the procedure of Stephens, and likewise the ML estimation is defined in the procedure with the BT.

Different constellations of distributions and parameters for $\mathrm{X}_{\mathrm{A}}$ are simulated and the estimations are tested. 10000 simulations were carried out for each constellation. $\mathrm{X}_{\mathrm{A}}$ was assumed as gamma distribution in each constellation. $\mathrm{X}_{\mathrm{A}}$ was simulated in each constellation by a simulation of a sample of the uniformly distributed random variable $\mathrm{U}$ and the using of the inverse function. Furthermore, a normal distributed sample was also calculated from the sample of $U$ with $\mu=0$ and $\sigma=1$. The parameters were estimated for this by using the ML method and the Anderson-Darling test is applied as well. The share of rejection for this procedure is the control value for the simulations.

The constellation with gamma distributed $\mathrm{X}_{\mathrm{A}}$ was not the only simulation. The logarithmic normal distribution for $X_{A}$ was simulated as well (the parameters are for $\ln \left(X_{A}\right)$ ). Furthermore the Burr distribution Type XII and the Pareto distribution were applied with

$$
\begin{gathered}
F(x)=1-\left[\eta /\left(\eta-x^{\tau}\right)\right]^{\lambda}, \quad x \geq 0, \eta>0, \tau>0, \lambda>0, \\
F(x)=(x+1)^{-\alpha}, \quad x \geq 0, \alpha>0 .
\end{gathered}
$$

These "false" distributions are used to validate the power of the test. The resulting shares of rejections for the different levels of significance are shown in Tables 2 to 4 for the sample sizes of $X_{A}$ of $n=25$, 50 and 100. The results of the Anderson-Darling test for a gamma distributed random variables are in the column "Classical Test". The real distribution of $\mathrm{X}_{\mathrm{A}}$ and their parameters are listed in the columns labelled as such. It is clear that the Anderson-Darling test with the BT works as well as the Anderson- 
Biased transformation and Goodness-of-Fit Test

Darling test with the procedure and the critical values of Stephens (1986) if the hypothesis $\mathrm{H}_{0}$ is right. The shares of rejections are very similar and nearly all are in the $90 \%$ range of the estimated share of a Bernoulli distribution. The ranges are $0.8 \%-1.1 \%$ for the level of $1 \%, 4.6 \%-5.3 \%$ for the level of $5 \%$ and $9.5 \%-10.4 \%$ for the level of $10 \%$. The shares of rejections are out of range in the case that $n=100$ and the level of significance are defined with 5\%. But in this constellation, the control value is also out of range.

The results of the classical test and the test with BT are very similar for the cases that $\mathrm{X}_{\mathrm{A}}$ is not gamma distributed. The differences between the classical test and the test with BT in the cases that $\mathrm{X}_{\mathrm{A}}$ are gamma distributed are small but partly significant. The new procedure with the BT reject more false assumptions if the sample size is not large $(n=25)$. The procedure according Stephens has a bit more power in the case of larger sample sizes $(n \geq 50)$. Independent of this fact - the new procedure works well and the power of the test is high. Both test procedures are not very powerful in the case that the real distribution is GPD and $\gamma$ is near 0 . The reason for this phenomena is that the GPD for $\gamma=0$ (Gumbel case) is an exponential distribution which is a special case of gamma distribution with $\alpha=1$. The GPDs with $\gamma$ near 0 and the gamma distributions with $\alpha$ near 1 are similar, no test can be very power full in this constellation.

\section{The Application of the BT for the Anderson-Darling Test for the Beta Distribution}

The only test specified for the beta distribution which was found in publications is an approach of $\mathrm{Li}$ and Papadopoulos (2002). The moments are used in this approach for goodness-of-fit tests for different distributions. But there is a mistake in the chapter for the beta distribution (a moment is divided by itself), and the power of the test is not so high for an exponential distribution. For example, $7-8 \%$ were rejected at a significance level of $5 \%$ in the case that $\mathrm{H}_{0}$ was correct ( $\mathrm{Li}$ and Papadopoulos, 2002, Tab. 1). This approach is not considered in this paper. Further tests or critical values for test specified for the beta distribution were not found in publications. In this place, the BT can be used for a first EDF test of goodness-of-fit for the beta distribution specified for it. The procedure includes the following steps:

- $\quad$ ML estimation of $\hat{\theta}_{\mathrm{A}}$ under the assumption of beta distribution for $\mathrm{X}_{\mathrm{A}}$. 
Biased transformation and Goodness-of-Fit Test

- Using this estimation of $\hat{\theta}_{\mathrm{A}}$ for $\theta_{\mathrm{B}}$.

- Definition of $\theta_{C}$ with $F_{C}$ as the CDF of a normal distribution with $\mu=0$ and $\sigma=1$.

- $\quad$ Computing the sample of $X_{\mathrm{D}}$ by the BT according equation 2.

- $\quad$ ML estimation of $\hat{\theta}_{D}$ of the sample of $\mathrm{X}_{\mathrm{D}}$, assumed as normal distribution $-\mathrm{H}_{0}$.

- $\quad$ Computing of the $\mathrm{A}^{2}$ for the sample of $\mathrm{X}_{\mathrm{D}}$ and test.

- Rejection of $\mathrm{H}_{0}$ at the defined level of significance if $\mathrm{A}^{* 2}>\mathrm{A}_{\text {critical }}^{2}$, otherwise acceptance of $\mathrm{H}_{0}$. The assumption that $\mathrm{X}_{\mathrm{A}}$ is beta distributed is rejected with the rejection of $\mathrm{H}_{0}$ that $\mathrm{X}_{\mathrm{D}}$ is normally distributed. The power function of this test has a poor quality even if the test works well. The test procedure is evaluated by simulations. Samples of beta distribution with variants of parameters were simulated (Tables 5-7). And distributions were simulated for $\mathrm{X}_{\mathrm{A}}$ which were not beta distributions. These were the log-normal distributions, the gamma distribution and the normal distribution. The lognormal and the gamma distributions were truncated at $\mathrm{x}=\mathrm{F}^{-1}(0.99)$ and scaled, so that $\mathrm{x}_{\max }=1$. The normal distribution was truncated with a lower bound $\mathrm{x}=\mathrm{F}^{-1}(0.01)$ and an upper bound $\mathrm{x}=\mathrm{F}^{-1}(0.99)$ and was located and scaled than so that $0 \leq \mathrm{x} \leq 1$.

The samples were simulated for the size of $n=10,25,50$ and 100. The considered levels of significance are $1 \%, 5 \%$ and $10 \%$. Furthermore, the $\chi^{2}$ test and the Kolomogorov-Smirnov test were applied for the sample sizes of $n=50$ and $n=100$ to compare the power of this test with the AndersonDarling test with the BT. The samples were divided in groups with 10 observations for the $\chi^{2}$ test. The bound between the groups was selected with the mean of the largest value of the smaller group and the minimum observation of the larger group. The variant of the Kolomogorov-Smirnov for a full specified distribution hypothesis was applied for the sample sizes of $n=50$ and $n=100$ likewise. The critical values of the $\chi^{2}$ test and the Kolomogorov-Smirnov test are taken from Rinne (2003, Chapter 3.4.5).

The shares of rejection from the 10000 simulations of each variant of real distribution are shown in Tables 5 through 7. The test procedure works well. The share of rejections for the case that $\mathrm{H}_{0}$ is true is almost equal to the defined level of significance. But some values in these cases are outside the $90 \%$ range for the estimation of the share of a Bernoulli distribution. The randomness of the simulation is reasonable for this because the control value is also often out of this range. The control value is again 
Biased transformation and Goodness-of-Fit Test

the share of rejections for the real normal distribution with estimated parameters for a normal distribution and the same underlying sample of $U$ used for the other real distributions as well. The Anderson-Darling test for the beta distribution by using the BT has more power than the $\chi^{2}$ test or the Kolomogorov-Smirnov test as shown in Tables 6 and 7. The $\chi^{2}$ test rejects less than the defined level of significance of beta distribution than the new procedure for the Anderson-Darling test in the cases that $\mathrm{H}_{0}$ is false; and the $\chi^{2}$ test rejects more than the defined level of significance of beta distribution. The error of the first kind is higher for the $\chi^{2}$ test than for the new procedure. The KolomogorovSmirnov test does not work; the power is very small.

\section{Practical application of tests for beta distribution}

The beta distribution is used for modeling of meteorological random variables. Yao (1974) modeled surface relative humidity of U.S. weather stations by using the beta distribution. Different data sets were analysed for example daily observations for one month. Parameters were estimated by the moment method. The goodness-of-fit was evaluated by using $\chi^{2}$-test. The number of observations per classes was not limited with $n_{i} \geq 10$ contrary to recommendations (Rinne, 2008, chapter 3.4.5.1). The fit is good according to this test results (Yao, 1974, Tab. 1).

The daily cloud duration was modeled by Chia and Hutchinson (1991) with beta distributions for different places in Australia. The goodness-of-fit was evaluated by a Kolmogorov-Smirnov test for a full specified distribution. The beta distribution can be used for most data according test results (Cia and Hutchinson, 1990, Tab. 3).

The beta distribution was applied furthermore for data of relative sunshine duration of Malaysia by Sulaiman et al. (1999). The goodness-of-fit was evaluated by the Kolmogorov-Smirnov test again. The fit is good for most of the data (Sulaiman et al., 1999, Tab. 2).

Neither the $\chi^{2}$-test nor the Kolmogorov-Smirnov test has a good performance for beta distribution as seen in Tables 6 and 7. Especially the Kolmogorov-Smirnov test has very low power. But till now there existed no better test. A practical example of meteorological data demonstrates the improvement of new test procedure for application of beta distribution. The data of Haarweg Wageningen weather station of Wageningen University (Netherlands) of July 2005 are analysed. The data set includes 
Biased transformation and Goodness-of-Fit Test

relative humidity. The relative sunshine duration is calculated by using sunshine minutes of data set and time between sunrise and sunset. The time between sunrise and sunset is assumed with linear function with $998.4 \mathrm{~min}$ for $1^{\text {st }}$ of July and $931.1 \mathrm{~min}$ for $31^{\text {st }}$ of July. Parameters of beta distribution are estimated with the ML method. The estimation is $\hat{\alpha}=7.47$ and $\beta=4.6255$ for relative humidity. The critical value for the Kolmogorov-Smirnov test is 0.23788 for $n=31$ and $5 \%$ significance and is not overstepped with $0.1253 . \mathrm{H}_{0}$ - beta distributed sample - is accepted. The $\chi^{2}$-test is applied with four classes with $n_{i}=7$ and 8 (although $n_{i}$ should be $\geq 10$ ). The critical value is $3.841(5 \%$, one grade of freedom) and is not overstepped by the test value of 2.686. Contrary to this the Anderson-Darling test value $\mathrm{A}^{* 2}=1.433$ and is much larger the $\mathrm{A}_{\text {crit }}{ }^{2}=0.753$ ( $5 \%$ significance). The sample is not beta distributed according to the Anderson-Darling test. The parameters of normal distribution of $X_{D}$ are $\hat{\mu}=-0.0005$ and $\hat{\sigma}=1.0164$. The results for data of relative sunshine duration per day are similar. Sample size is $n=31$ again. Parameters are $\hat{\alpha}=1.5536$ and $\beta=0.7480$. The value of the Kolmogorov-Smirnov test is 0.1706 and doesn't overstep critical value. The value of $\chi^{2}$-test is 3.9937 . This oversteps the critical value a bit. $\mathrm{H}_{0}$ would be rejected according to the $\chi^{2}$-test but not according to the KolmogorovSmirnov test. The Anderson-Darling test rejects the hypothesis of beta distributed data very clearly with $A^{* 2}=2.059$. The parameters of the normal distribution of $X_{D}$ are $\hat{\mu}=-0.0116$ and $\hat{\sigma}=1.0057$. The estimated CDF and observed data are shown in Fig. 3. The fit is not good.

The example shows that the introduced procedure of the Anderson-Darling test for beta distribution by using BT is an improvement of applied statistic. It seems that many data are assumed likely as beta distributed in pasted analysis although they are not beta distributed.

\section{Summary and Discussion}

The BT is introduced and can be used exactly for each of the GEV, the GPD and the normal distribution. The BT works as an approximation for the gamma, beta and normal distribution as a family of distributions. Because of this the Anderson-Darling Test for normal distributions can be used as a goodness-of-fit test for the gamma and the beta distribution. The new test procedure works with similar power as the classical procedure of Stephens. The power of the new procedure is a bit higher 
Biased transformation and Goodness-of-Fit Test

for smaller sample sizes. The classical procedure has a bit more power for larger sample sizes. The same test procedure in principle is used for a first goodness-of-fit test specified for beta distribution. This is the first test specified for the beta distribution. The power function of the new test is poor because of the basics of the BT. And it is defined in the test procedure that the ML estimated parameters of assumed the gamma or beta distribution has to be used. The method of parameter estimation is defined in the procedures of Stephens. The general question is: Is it necessary to have a good power function for the whole range of parameters for a powerful test if the estimation method is defined in the test procedure? A good power function is not necessary according to the results of this paper. The Anderson-Darling test for the gamma distribution with the new procedure has a similar power as the classical Anderson-Darling test for the gamma distribution. And the power function of the new procedure is very poor compared with the function of the classical test. The poor power function can result only in wrong test results, if the test procedure is not used right and the parameter vector $\theta_{\mathrm{A}}$ is not estimated correct with the ML method.

If the new test does not reject the hypothesis, the user can assume that the ML estimation of the parameters leads to an estimated distribution with similar goodness-of-fit as for a sample of normal distribution with the equal underlying sample of $U$ as for the gamma or beta distribution. It was shown in the validation of the BT that the goodness-of-fit of a beta, gamma or normal distributed sample from the same underlying sample of $U$ is almost equal (Figures $2 \mathrm{c}$ and $\mathrm{d}$ ).

The CDF of the normal, gamma and beta distributions are computed by using approximations from different software libraries (Excel was used and the library of www.extremeoptimization.com). These approximations work well in the common ranges of parameters and percentiles. But if, for example, $\alpha$ of the gamma distribution is small and the percentile is as well, the approximation is wrong. The BT of the normal, gamma and beta distribution cannot work better than the approximation of the CDF of these distributions. This is a reason why larger simulations were not carried out for the validation of the BT for normal, gamma and beta distribution. The probability that the approximation of the CDF does not work in a variant of parameters and sample of $U$ increases with the number of simulations. The failure of the approximation is avoided by a limitation of the simulations. And more simulations 
Biased transformation and Goodness-of-Fit Test

are not necessary, because the distributions of the values of goodness-of-fit in the validation of the BT were tested with knowledge of the simulation size.

The other EDF-tests specified for the normal distribution could be used in the test procedure with the BT too. The power of such tests could be evaluated in further works. The Anderson-Darling test has the better quality in the test of normality (Landry and Lepage, 1992). That is why only this EDF-test is applied in this paper.

The practical relevance of the new test for beta distributions was demonstrated successfully in metrological data.

The BT is interesting irrespective of the introduced application for the test of the gamma and beta distribution. Perhaps the BT will become a criterion for the classification of distributions or the BT can perhaps be used for estimations of the GPD or GEV or for their tests.

The possibility of $\mathrm{BT}$ to estimate the PDF or CDF of a beta distribution by using the normal distribution should be researched in the future.

\section{Acknowledgement}

The author would like to thank the Federal Ministry for Education and Science of Germany for the financial support of the FloodRisk project in the EraNet Crue initiative; the research for the paper was partly carried out within this project. Furthermore I would like to express my gratitude to Michael

\section{A. Stephens for the helpful communication.}

\section{References}

Anderson, T.W., Darling, D. A. (1952). Asymptotic theory of certain "Goodness-of-fit" criteria based on stochastic processes. Ann. Math. Statist. 23: 193-212.

Anderson, T.W., Darling, D. A. (1954). A test for goodness-of-fit. J. Amer. Statist. Assoc. 49: 300-310

Birnbaum, Z.W., Hall, R.A. (1960). Small Sample Distributions for Multi-Sample Statistics of Smirnov Type. Ann. Math. Statist. 31: 710-720

Bowman, K.O., Shenton, L.R. (1986). Moment ( $\left.\sqrt{ }_{1}, b_{2}\right)$ Techniques. In: D’Augustino, R.B., Stephens, M.A. ed., Goodness-of-Fit Techniques. Statistics: textbooks and monographs, Vol. 68: 279-327, Marcel Dekker: New York

Box, G.E.P.; Cox, D.R. (1964). An Analysis of Transformations. J. R. Statist. Soc. B, 26: 211-246 
Biased transformation and Goodness-of-Fit Test

Carley, H., Taylor, M.D. (2000). A new proof of Sklar's theorem. Conference on Distributions with given marginals, Barcelona, July 2000

Chia, E., Hutchinson, M.F. (1991). The beta distribution as a probability model for daily cloud duration. Agriculture and Forest Meteorology, 56, 195-208

Johnson, N.L., Kotz, S., Balakrishnan, N. (1994). Continous Univariate Distributions- Volume 1. 2nd Ed. Wiley: New York

Johnson, N.L., Kotz, S., Balakrishnan, N. (1995). Continous Univariate Distributions- Volume 1. 2nd Ed. Wiley: New York

Kolmogorov, A.N. (1933). Sulle determinazione empirica di una legged i distibuziane. Giorna. Ist. Attuari. 4: 83-91

Kuiper, N.H. (1960). Tests concerning random points on a circle. Proc. Konink. Neder. Akad. Van. Wetenschappen, A 63: 38-47.

Landry, L., Leparge, Y. (1992). Empirical Behavior of some tests for Normality. Commun. Statist. B - Computa. Simula. 21: 971-999

Li, G., Papadopoulos, A. (2002). A note on Goodness of fit test using moments. STATISTICA, 62, n. 1: 72-86

Moore, D.S. (1986). Tests of Chi-Squared Type. In: D’Augustino, R.B., Stephens, M.A. ed., Goodness-of-Fit Techniques. Statistics: textbooks and monographs, Vol. 68: 63-95, Marcel Dekker: New York

Quesenberry, C.P. (1986). Some transformation methods in Goodness of fit. In: D'Augustino, R.B., Stephens, M.A. ed., Goodness-of-Fit Techniques. Statistics: textbooks and monographs, Vol. 68: 235-278, Marcel Dekker, New York

Rinne, H. (2003). Taschenbuch der Statistik (German; Handbook of the statistic). Harri Deutsch, Frankfurt

Sklar, M. (1959). Fonctions de repartition à N dimensions et leurs marges. Publ. Inst. Stat. Paris, 8: 229-231

Stephens, M.A. (1974). EDF Statistics for Goodness of Fit and Some Comparisons. J. Am. Statist. Assoc. 69, No 347: $730-737$

Stephens, M.A. (1976). Asymptotic results for Goodness-of-Fit statistics with unknown parameters. The Annals of Statistics 4, no2: 357-369

Stephens, M.A. (1986). Tests based on EDF statistics. In: D’Augustino, R.B., Stephens, M.A. ed., Goodness-ofFit Techniques. Statistics: textbooks and monographs, Vol. 68: 97-194, Marcel Dekker: New York Sulaiman, M.Y., Oo, H.W.M., Wahab, M.A., Zakaria, A. (1999). Application of Beta distribution model to Malaysian sunshine data. Renewable Energy 18: 573-579 
Biased transformation and Goodness-of-Fit Test

Yao, A.Y.M. (1974). A Statistical Model for the Surface relative humidity. Journal of Applied Meteorology. Val. 13: $17-21$

Wageningen University, Netherlands, Meteorology and Air Quality section (2009, download). Weather station Haarweg Wageningen. Data: http://www.met.wau.nl/

Watson, G.S. (1961). Goodness-of-fit tests on a circle. Biometrika 48: 109-114.

\section{Appendix}

\section{The BT for the GEV}

The GEV is formulated for the Gumbel case

$$
F(x)=\exp \{-\exp [-(x-\mu) / \sigma]\}
$$

and is formulated for the Fréchet and Weibull case with

$$
F(x)=\exp \left\{-[1+\gamma(x-\mu) / \sigma]^{-1 / \gamma}\right\}
$$

where the parameter is $\sigma>0$. The lower bound is for the Fréchet case $\gamma>0$

$$
x \geq \mu-\sigma / \gamma \text {. }
$$

The upper bound is for the Weibull case with $\gamma<0$

$$
x \leq \mu-\sigma / \gamma .
$$

Theorem A1: If $X_{A}$ is GEV-Gumbel distributed and $F_{B}$ is the CDF of a GED-Gumbel and $F_{C}$ is the $C D F$ of a GEV-Fréchet/Weibull with any parameters, then $X_{A}$ can be transformed to $X_{D}$ with $X_{D}=F_{C}$ ${ }^{1}\left(F_{B}\left(X_{A}\right)\right)$ and $X_{D}$ is GEV-Fréchet/Weibul distributed with the parameters

$$
\begin{gathered}
\gamma_{D}=\gamma_{C} \sigma_{A} / \sigma_{B}, \\
\sigma_{D}=\sigma_{A} \sigma_{C} \exp \left[\gamma_{C}\left(\mu_{A}-\mu_{B}\right) / \sigma_{B}\right] / \sigma_{B}, \\
\mu_{D}=\sigma_{C} \exp \left[\gamma_{C}\left(\mu_{A}-\mu_{B}\right) / \sigma_{B}\right] / \gamma_{C}-\sigma_{C} / \gamma_{C}+\mu_{C}
\end{gathered}
$$

and the transformation function $x_{D}\left(x_{A}\right)$ is

$$
x_{D}=\left\{\sigma_{C} \exp \left[\gamma_{C}\left(x_{A}-\mu_{B}\right) / \sigma_{B}\right]-\sigma_{C}+\gamma_{C} \mu_{C}\right\} / \gamma_{C} .
$$

Proof. If equations 8-11 are used in $\mathrm{F}_{\mathrm{D}}\left(\mathrm{x}_{\mathrm{D}}\right)$ according to equation 7 , then according to equation 6 the $\mathrm{CDF} \mathrm{F}_{\mathrm{A}}\left(\mathrm{x}_{\mathrm{A}}\right)$ can be formulated after simplifications so that equation 3 is valid.

This transformation works likewise the other way round if the bounds of $X_{D}$ and $X_{C}$ are the same with

$$
\mu_{C}-\sigma_{C} / \gamma_{C}=\mu_{D}-\sigma_{D} / \gamma_{D}
$$

and $\gamma_{C}$ and $\gamma_{D}$ have the same sign.

Theorem A2: If $X_{A}$ is GEV-Gumbel distributed and $F_{B}$ is the CDF of a GEV-Gumbel and $F_{C}$ is the $C D F$ of a GEV-Gumbel with any parameters, then $X_{A}$ can be transformed to $X_{D}$ with $X_{D}=F_{C}^{-1}\left(F_{B}\left(X_{A}\right)\right)$ and $X_{D}$ is GEV-Gumbel distributed with the parameters

$$
\begin{gathered}
\sigma_{D}=\sigma_{A} \sigma_{C} / \sigma_{B} . \\
\mu_{D}=\left(-\mu_{B} \sigma_{C}+\mu_{C} \sigma_{B}+\mu_{A} \sigma_{C}\right) / \sigma_{B}
\end{gathered}
$$

and the transformation function $x_{D}\left(x_{A}\right)$ is

$$
x_{D}=\left(\sigma_{B} \mu_{C}+\sigma_{C} x_{A}-\mu_{B} \sigma_{C}\right) / \sigma_{B} .
$$


Biased transformation and Goodness-of-Fit Test

Proof: If equations 12-14 are used in $\mathrm{F}_{\mathrm{D}}\left(\mathrm{x}_{\mathrm{D}}\right)$ according to equation 6, then according to equation 6 the function $\mathrm{F}_{\mathrm{A}}\left(\mathrm{x}_{\mathrm{A}}\right)$ can be formulated after simplifications so that equation 3 is valid.

Theorem A3: If $X_{A}$ is GEV-Fréchet/Weibull distributed and $F_{B}$ is the CDF of a GEV-Fréchet/Weibull and $F_{C}$ is the $C D F$ of a GEV-Fréchet/Weibull with any parameters but with

$$
\sigma_{A} / \gamma_{A}-\mu_{A}=\sigma_{B} / \gamma_{B}-\mu_{B}
$$

and $\gamma_{A}$ and $\gamma_{B}$ have the same sign then $X_{A}$ can be transformed to $X_{D}$ with $X_{D}=F_{C}^{-1}\left(F_{B}\left(X_{A}\right)\right)$ and $X_{D}$ is GEV-Fréchet/Weibull distributed with the parameters

$$
\begin{gathered}
\gamma_{D}=\gamma_{A} \gamma_{C} / \gamma_{B}, \\
\sigma_{D}=\left[\sigma_{A} \gamma_{B} /\left(\gamma_{A} \sigma_{B}\right)\right]^{\gamma / \gamma_{B}} \sigma_{C} \gamma_{A} / \gamma_{B}, \\
\mu_{D}=\mu_{C}-\sigma_{C} / \gamma_{C}+\left[\sigma_{A} \gamma_{B} /\left(\gamma_{A} \sigma_{B}\right)\right]^{\gamma_{C} / \gamma_{B}} \sigma_{C} / \gamma_{C}
\end{gathered}
$$

and the transformation function $x_{D}\left(x_{A}\right)$ is

$$
x_{D}=\left\{\sigma_{C}\left[1+\gamma_{B}\left(x_{A}-\mu_{B}\right) / \sigma_{B}\right]^{\gamma_{C} / \gamma_{B}}-\sigma_{C}+\gamma_{C} \mu_{C}\right\} / \gamma_{C} .
$$

Proof: If equations 16-19 are used in $\mathrm{F}_{\mathrm{D}}\left(\mathrm{x}_{\mathrm{D}}\right)$ according to equation 7 , and equation 15 is considered, then according equation 7 the function $\mathrm{F}_{\mathrm{A}}\left(\mathrm{x}_{\mathrm{A}}\right)$ can be formulated after some simplifications so that equation 3 is valid.

\section{The BT for the GPD}

The GPD is formulated for the Gumbel case

$$
F(x)=1-\exp (-x / \sigma)
$$

and for the Fréchet and Weibull case formulated with

$$
F(x)=1-(1+\gamma x / \sigma)^{-1 / \gamma} .
$$

Generally $x \geq 0$ und $\sigma>0$. There is an upper bound for the Weibull case $\gamma<0$ with

$$
x \leq-\sigma / \gamma \text {. }
$$

Theorem A4. If $X_{A}$ is GPD-Gumbel distributed and $F_{B}$ is the CDF of a GPD-Gumbel and $F_{C}$ is the $C D F$ of a GPD-Gumbel with any parameters, then $X_{A}$ can be transformed to $X_{D}$ with $X_{D}=F_{C}^{-1}\left(F_{B}\left(X_{A}\right)\right)$ and $X_{D}$ is GPD-Gumbel distributed with the parameters

$$
\sigma_{D}=\sigma_{A} \sigma_{C} / \sigma_{B}
$$

and the transformation function $x_{D}\left(x_{A}\right)$ is

$$
x_{D}=x_{A} \sigma_{C} / \sigma_{B} .
$$

Proof: If equations 22-23 are used in $\mathrm{F}_{\mathrm{D}}\left(\mathrm{x}_{\mathrm{D}}\right)$ according equation 20, then according to equation 20 the function $\mathrm{F}_{\mathrm{A}}\left(\mathrm{x}_{\mathrm{A}}\right)$ can be formulated after some simplifications so that equation 3 is valid.

Theorem A5: If $X_{A}$ is GPD-Fréchet/Weibull distributed and $F_{B}$ is the CDF of a GPD-Fréchet/Weibull and $F_{C}$ is the CDF of a GPD-Fréchet/Weibull with any parameters but with

$$
\sigma_{A} / \gamma_{A}=\sigma_{B} / \gamma_{B}
$$

then $X_{A}$ can be transformed to $X_{D}$ with $X_{D}=F_{C}^{-1}\left(F_{B}\left(X_{A}\right)\right)$ and $X_{D}$ is GPD-Fréchet/Weibull distributed with the parameters

$$
\begin{gathered}
\gamma_{D}=\gamma_{A} \gamma_{C} / \gamma_{B}, \\
\sigma_{D}=\gamma_{A} \sigma_{C} / \gamma_{B}
\end{gathered}
$$

and the transformation function $x_{D}\left(x_{A}\right)$ is

$$
x_{D}=\sigma_{C}\left\{\left(1+\gamma_{B} x_{A} / \sigma_{B}\right)^{\left.\gamma_{C} / \gamma_{B}-1\right\} / \gamma_{C}} .\right.
$$


Biased transformation and Goodness-of-Fit Test

Proof: If equations 25-27 are used in $\mathrm{F}_{\mathrm{D}}\left(\mathrm{x}_{\mathrm{D}}\right)$ according to equation 21 under consideration of equation 24 , then according to equation 21 , the function $\mathrm{F}_{\mathrm{A}}\left(\mathrm{x}_{\mathrm{A}}\right)$ can be formulated after some simplifications so that equation 3 is valid.

The condition of equation 24 has to be also considered for the Fréchet case even this is not a real bound of X.

Theorem A6: If $X_{A}$ is GPD-Gumbel distributed and $F_{B}$ is the CDF of a GPD-Gumbel and $F_{C}$ is the $C D F$ of a GPD-Fréchet/Weibull with any parameters, then $X_{A}$ can be transformed to $X_{D}$ with $X_{D}=F_{C}$ ${ }^{I}\left(F_{B}\left(X_{A}\right)\right)$ and $X_{D}$ is GPD-Fréchet/Weibull distributed with the parameters

$$
\begin{gathered}
\gamma_{D}=\gamma_{C} \sigma_{A} / \sigma_{B}, \\
\sigma_{D}=\sigma_{A} \sigma_{C} / \sigma_{B}
\end{gathered}
$$

and the transformation function $x_{D}\left(x_{A}\right)$ is

$$
x_{A}=\sigma_{C}\left\{\exp \left(x_{A} \gamma_{C} / \sigma_{B}\right)-1\right\} / \gamma_{C} .
$$

Proof: If equations 28-30 are used in $\mathrm{F}_{\mathrm{D}}\left(\mathrm{x}_{\mathrm{D}}\right)$ according to equation 20, then according to equation 21, the function $\mathrm{F}_{\mathrm{A}}\left(\mathrm{x}_{\mathrm{A}}\right)$ can be formulated after some simplifications so that equation 3 is valid.

It is possible to transform $\mathrm{X}_{\mathrm{D}}$ to $\mathrm{X}_{\mathrm{A}}$ with any $\mathrm{F}_{\mathrm{C}}(\mathrm{x})$ and $\mathrm{F}_{\mathrm{B}}(\mathrm{x})$. It has only to be ensured that

$$
\sigma_{C} / \gamma_{C}=\sigma_{D} / \gamma_{D}
$$

\section{The Normal Distribution}

Theorem A7: If $X_{A}$ is normally distributed and $F_{B}$ and $F_{C}$ are $C D F$ of normal distributions with any parameters, then $X_{A}$ can be transformed to $X_{D}$ with $X_{D}=F_{C}^{-1}\left(F_{B}\left(X_{A}\right)\right)$ and $X_{D}$ is normally distributed with the parameters

$$
\begin{gathered}
\mu_{D}=\mu_{C}+\sigma_{C}\left(\mu_{A}-\mu_{B}\right) / \sigma_{B}, \\
\sigma_{D}=\sigma_{A} \sigma_{D} / \sigma_{C}
\end{gathered}
$$

and the transformation function $x_{D}\left(x_{A}\right)$ is

$$
x_{D}=\mu_{C}+\sigma_{C}\left(x_{A}-\mu_{B}\right) / \sigma_{B}
$$

Proof: If equation 4 is taken and the PDF are replaced by adaptations of equation 5 it can be simplified

$$
f_{D}\left(x_{D}\right)=\frac{\sigma_{B}}{\sigma_{A} \sigma_{C}} \frac{\exp \left\{\left[x_{A}\left(x_{D}\right)-\mu_{A}\right]^{2} /\left(2 \sigma_{A}^{2}\right)\right\} \exp \left[-\left(x_{D}-\mu_{C}\right)^{2} /\left(2 \sigma_{C}^{2}\right)\right]}{\sqrt{2 \pi} \exp \left\{\left[x_{A}\left(x_{D}\right)-\mu_{B}\right]^{2} /\left(2 \sigma_{B}^{2}\right)\right\}} .
$$

With equation 32, for the replacing of $\sigma_{\mathrm{A}}$ it can be modified and written

$$
f_{D}\left(x_{D}\right)=1 /\left(\sigma_{D} \sqrt{2 \pi}\right) \exp \left\{\left[x_{A}\left(x_{D}\right)-\mu_{A}\right]^{2} \sigma_{C}^{2} /\left(2 \sigma_{D}^{2} \sigma_{B}^{2}\right)\right\} \exp \left\{\left(x_{D}-\mu_{C}\right)^{2} / \sigma_{C}^{2}+\left[x_{A}\left(x_{D}\right)-\mu_{B}\right]^{2} / \sigma_{B}^{2}\right\}^{0.5}
$$

Further $\mathrm{x}_{\mathrm{A}}\left(\mathrm{x}_{\mathrm{D}}\right)$ can be replaced by using equation 33, and it can be simplified and modified to

$$
f_{D}\left(x_{D}\right)=1 /\left(\sigma_{D} \sqrt{2 \pi}\right) \exp \left\{\left[\sigma_{B} / \sigma_{C}\left(x_{D}-\mu_{C}\right)+\mu_{B}-\mu_{A}^{2} \sigma_{C}^{2}\right] /\left(2 \sigma_{D}^{2} \sigma_{B}^{2}\right)\right\} .
$$

The parameter $\mu_{\mathrm{D}}$ can be replaced by the using of equation 31. It can be written

$$
f_{D}\left(x_{D}\right)=1 /\left(\sigma_{D} \sqrt{2 \pi}\right) \exp \left[-\left\{\sigma_{B} / \sigma_{C}\left(x_{D}-\mu_{C}\right)+\mu_{B}-\mu_{D} \sigma_{B} / \sigma_{C}+\mu_{C} \sigma_{B} / \sigma_{C}-\mu_{B}\right\}^{2} \sigma_{C}^{2} / 2 / \sigma_{D}^{2} / \sigma_{B}^{2}\right\rfloor
$$

and simplified to

$$
f_{D}\left(x_{D}\right)=1 /\left(\sigma_{D} \sqrt{2 \pi}\right) \exp \left\{-\left(x_{D}-\mu_{D}\right)^{2} 2 / \sigma_{D}^{2}\right\}
$$

which is the PDF for $\mathrm{X}_{4}$ according to equation $5 . \mathrm{X}_{4}$ is normally distributed.

\section{Figures and Tables}


Biased transformation and Goodness-of-Fit Test

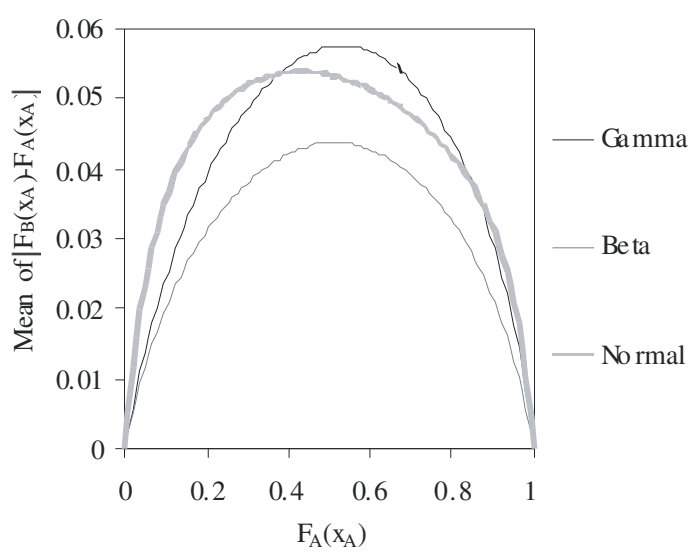

a)

Figure 1: Bias of the BT for the simulation of $n=1000$ for the different types of $F_{A}$ and $F_{B}$ : a) mean of the absolute difference $\left.\left|\mathrm{F}_{\mathrm{B}}\left(\mathrm{x}_{\mathrm{A}}\right)-\mathrm{F}_{\mathrm{A}}\left(\mathrm{x}_{\mathrm{A}}\right)\right|, \mathrm{b}\right)$ extremes of $\mathrm{F}_{\mathrm{B}}\left(\mathrm{x}_{\mathrm{A}}\right)$ in relation to $\mathrm{F}_{\mathrm{A}}\left(\mathrm{x}_{\mathrm{A}}\right)$

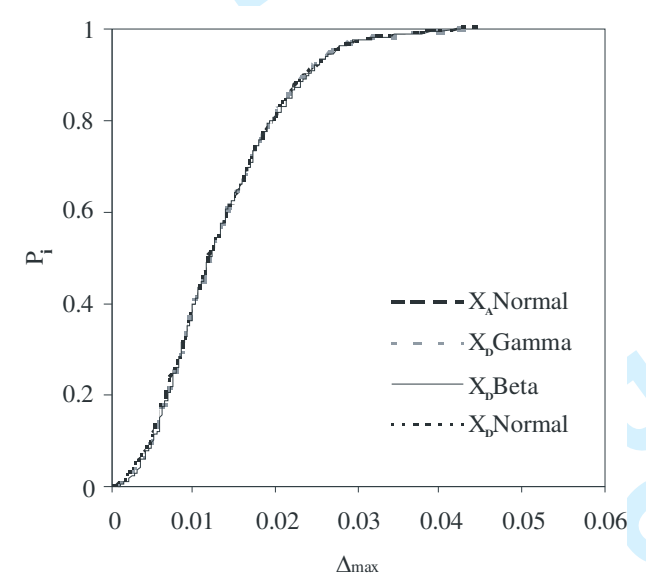

a)

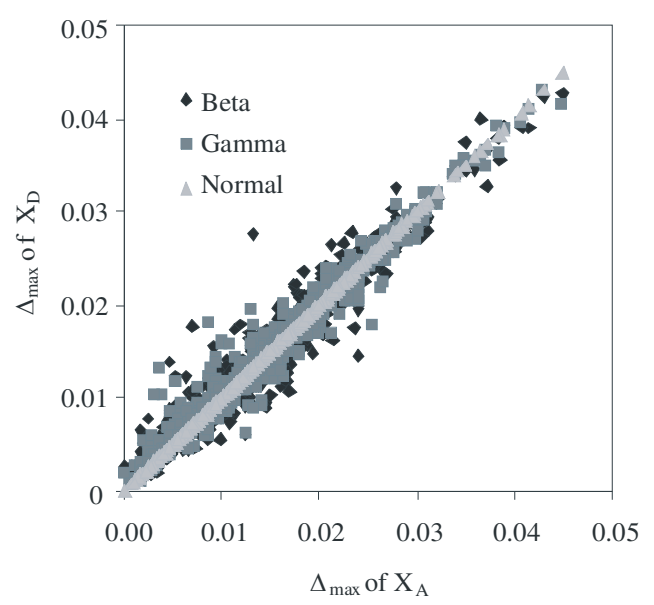

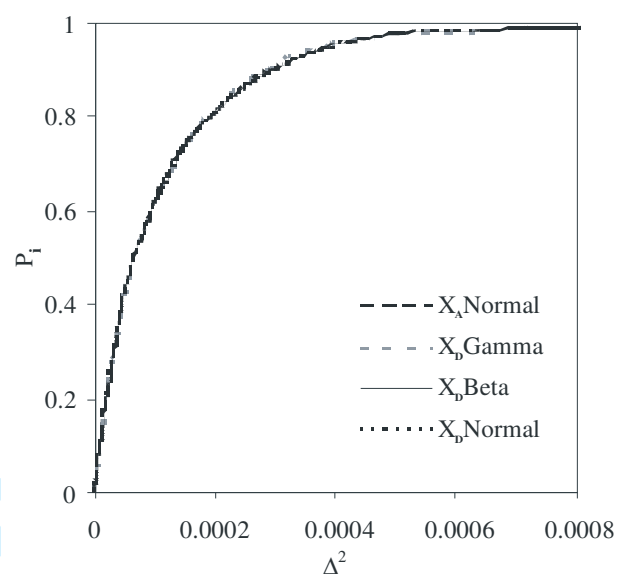

b)

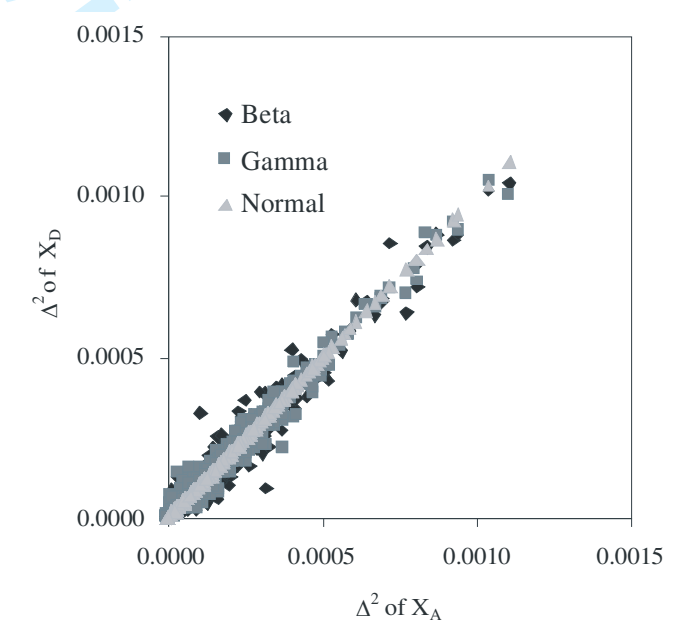

d)

)

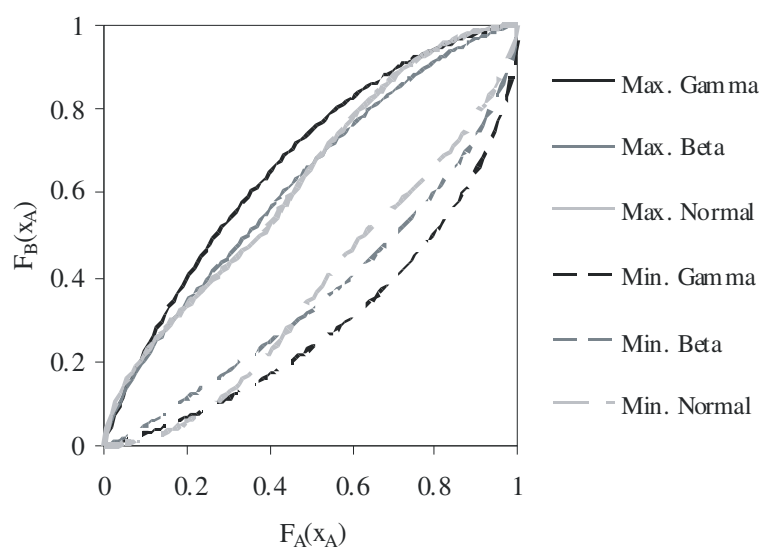

)

Figure 2: Results of the simulations for $\mathrm{n}=1000$ with $\mathrm{X}_{\mathrm{A}}$ as normally distributed and the different distribution types of $\mathrm{X}_{\mathrm{D}}$ : a) distributions of $\Delta_{\max }$, b) distributions of $\Delta^{2}$, c) $\Delta_{\max }$ of $\mathrm{X}_{\mathrm{A}}$ to $\Delta_{\max }$ of $\mathrm{X}_{\mathrm{D}}$, d) $\Delta^{2}$ of $\mathrm{X}_{\mathrm{A}}$ to $\Delta^{2}$ of $\mathrm{X}_{\mathrm{D}}$ 
Biased transformation and Goodness-of-Fit Test

1

2

3

4

5

6

7

8

9

10

11

12

13

14

15

16

17

18

19

20

21

22

23

24

25

26

27

28

29

30

31

32

33

34

35

36

37

38

39

40

41

42

43

44

45

46

47

48

49

50

51

52

53

54

55

56

57

58

59

60 a)

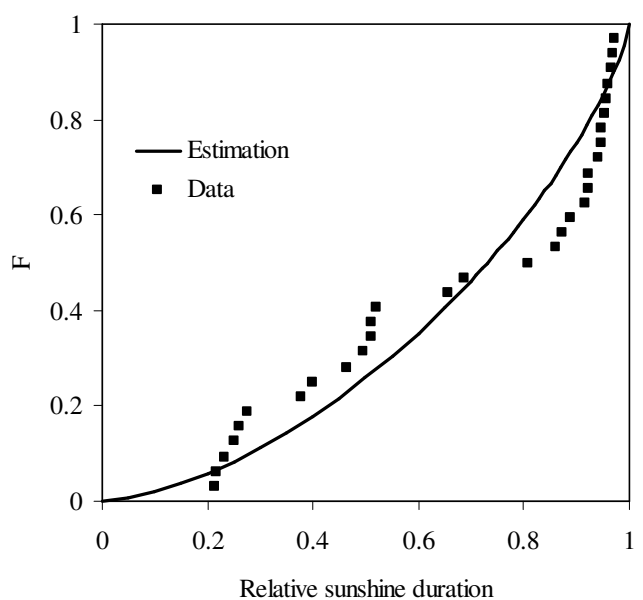

Figure 3: Applied beta distribution: a) relative sunshine duration, b) relative humidity 
Biased transformation and Goodness-of-Fit Test

Table 1: Test values of the Kolomogorov-Smirnov test for homogeneity for the distributions of $\Delta_{\max }$ and $\Delta^{2}$ for the different variants of sample size, distribution of $\mathrm{X}_{\mathrm{A}}$ and distribution of $\mathrm{X}_{\mathrm{D}}$

\begin{tabular}{|c|c|c|c|c|c|}
\hline \multirow[t]{2}{*}{ Sample size } & \multirow[t]{2}{*}{ Distribution of $\mathbf{X}_{\mathrm{A}}$} & \multirow[t]{2}{*}{ Distribution of Value } & \multicolumn{3}{|c|}{ Distribution of $X_{D}$} \\
\hline & & & Gamma & Beta & Normal \\
\hline \multirow{6}{*}{$\mathrm{n}=10$} & Gamma & $\Delta_{\max }$ & 0.028 & 0.030 & 0.008 \\
\hline & & $\Delta^{2}$ & 0.021 & 0.019 & 0.005 \\
\hline & Beta & $\Delta_{\max }$ & 0.009 & 0.008 & 0.030 \\
\hline & & $\Delta^{2}$ & 0.027 & 0.026 & 0.005 \\
\hline & Normal & $\Delta_{\max }$ & 0.030 & 0.031 & 0.001 \\
\hline & & $\Delta^{2}$ & 0.033 & 0.036 & 0.001 \\
\hline \multirow{6}{*}{$\mathrm{n}=100$} & Gamma & $\Delta_{\max }$ & 0.013 & 0.016 & 0.010 \\
\hline & & $\Delta^{2}$ & 0.018 & 0.017 & 0.010 \\
\hline & Beta & $\Delta_{\max }$ & 0.015 & 0.018 & 0.010 \\
\hline & & $\Delta^{2}$ & 0.014 & 0.019 & 0.009 \\
\hline & Normal & $\Delta_{\max }$ & 0.016 & 0.021 & 0.001 \\
\hline & & $\Delta^{2}$ & 0.018 & 0.016 & 0.001 \\
\hline \multirow{6}{*}{$\mathrm{n}=1000$} & Gamma & $\Delta_{\max }$ & 0.016 & 0.019 & 0.017 \\
\hline & & $\Delta^{2}$ & 0.016 & 0.017 & 0.015 \\
\hline & Beta & $\Delta_{\max }$ & 0.022 & 0.020 & 0.016 \\
\hline & & $\Delta^{2}$ & 0.021 & 0.019 & 0.023 \\
\hline & Normal & $\Delta_{\max }$ & 0.019 & 0.020 & 0.001 \\
\hline & & $\Delta^{2}$ & 0.016 & 0.026 & 0.001 \\
\hline
\end{tabular}

Table 2: Percentage or rejection of $\mathrm{H}_{0}$ of the classical Anderson-Darling test, the test with the BT for gamma distribution for different significance levels and $n=25$. The level of significance is written in percentage.

\begin{tabular}{cccccccc}
\hline $\begin{array}{c}\text { Real } \\
\text { Distribution }\end{array}$ & Parameters & \multicolumn{3}{c}{ Classical Test } & \multicolumn{3}{c}{ Test with the BT } \\
\hline LogNorm & $\mu=1, \sigma^{2}=0.25$ & 3.42 & 10.92 & 18.19 & 3.69 & 11.41 & 18.89 \\
LogNorm & $\mu=1, \sigma^{2}=1$ & 13.26 & 27.97 & 38.08 & 13.43 & 27.98 & 38.42 \\
BurrXII & $\eta=4, \tau=8, \lambda=1$ & 4.74 & 12.1 & 19.41 & 5.28 & 13.32 & 21.08 \\
BurrXII & $\eta=1, \tau=1, \lambda=2$ & 14.62 & 27.49 & 36.67 & 13.95 & 27.2 & 36.46 \\
Pareto & $\alpha=8$ & 1.52 & 6.63 & 12.02 & 1.68 & 6.93 & 12.68 \\
Pareto & $\alpha=2$ & 14.62 & 27.49 & 36.67 & 13.95 & 27.2 & 36.46 \\
GPD & $\sigma=1, \gamma=-1 / 16$ & 0.91 & 4.67 & 9.56 & 0.92 & 4.9 & 10.01 \\
GPD & $\sigma=1, \gamma=-1 / 4$ & 1.71 & 7.68 & 14.06 & 1.73 & 7.57 & 13.73 \\
Gamma & $\lambda=1 . \alpha=0.5$ & 0.86 & 4.62 & 9.46 & 0.86 & 4.87 & 9.72 \\
Gamma & $\lambda=1 . \alpha=1$ & 0.87 & 4.84 & 9.58 & 0.97 & 5.09 & 10.05 \\
Gamma & $\lambda=1 . \alpha=2.5$ & 0.89 & 4.92 & 9.72 & 0.98 & 5.18 & 10.07 \\
Gamma & $\lambda=1 . \alpha=5$ & 0.89 & 4.89 & 9.69 & 0.97 & 5.18 & 10.14 \\
Gamma & $\lambda=1 . \alpha=10$ & 0.89 & 4.88 & 9.72 & 0.98 & 5.17 & 10.13 \\
Control & $\mu=0, \sigma=0$ & - & - & - & 0.97 & 5.18 & 10.09 \\
\hline
\end{tabular}


Biased transformation and Goodness-of-Fit Test

Table 3: Percentage or rejection of $\mathrm{H}_{0}$ of the classical Anderson-Darling test, the test with the BT for gamma distribution for different significance levels and $\mathrm{n}=50$. The level of significance is written in percentage.

\begin{tabular}{cccccccc}
\hline \multirow{2}{*}{$\begin{array}{c}\text { Real } \\
\text { Distribution }\end{array}$} & Parameters & \multicolumn{3}{c}{ Classical Test } & \multicolumn{3}{c}{ Test with the BT } \\
& & $1 \%$ & $5 \%$ & $10 \%$ & $1 \%$ & $5 \%$ & $10 \%$ \\
\hline LogNormal & $\mu=1, \sigma^{2}=0.25$ & 6.68 & 18.23 & 27.29 & 6.82 & 18.26 & 27.51 \\
LogNormal & $\mu=1, \sigma^{2}=1$ & 33.24 & 53.23 & 63.57 & 31.46 & 51.51 & 62.1 \\
BurrXII & $\eta=4, \tau=8, \lambda=1$ & 7.74 & 18.36 & 26.82 & 8.5 & 19.77 & 28.22 \\
BurrXII & $\eta=1, \tau=1, \lambda=2$ & 34.32 & 50.4 & 59.21 & 31.19 & 47.81 & 56.89 \\
Pareto & $\alpha=8$ & 2.66 & 8.43 & 14.56 & 2.46 & 8.26 & 14.44 \\
Pareto & $\alpha=2$ & 34.32 & 50.4 & 59.21 & 31.19 & 47.81 & 56.89 \\
GPD & $\sigma=1, \gamma=-1 / 16$ & 0.98 & 4.95 & 9.75 & 1 & 4.84 & 9.71 \\
GPD & $\sigma=1, \gamma=-1 / 4$ & 3.2 & 11.59 & 20.09 & 3.04 & 10.94 & 18.81 \\
Gamma & $\lambda=1 . \alpha=0.5$ & 0.96 & 4.67 & 9.51 & 0.95 & 4.64 & 9.61 \\
Gamma & $\lambda=1 . \alpha=1$ & 0.97 & 4.68 & 9.4 & 1.04 & 4.72 & 9.61 \\
Gamma & $\lambda=1 . \alpha=2.5$ & 0.97 & 4.7 & 9.51 & 1.06 & 4.83 & 9.65 \\
Gamma & $\lambda=1 . \alpha=5$ & 0.97 & 4.69 & 9.49 & 1.05 & 4.81 & 9.59 \\
Gamma & $\lambda=1 . \alpha=10$ & 1 & 4.67 & 9.46 & 1.05 & 4.84 & 9.57 \\
Control & $\mu=0, \sigma=0$ & - & - & - & 1.03 & 4.83 & 9.54 \\
\hline & & & & & & &
\end{tabular}

Table 4: Percentage or rejection of $\mathrm{H}_{0}$ of the classical Anderson-Darling test, the test with the BT for gamma distribution for different significance levels and $n=100$. The level of significance is written in percentage.

\begin{tabular}{cccccccc}
\hline \multirow{2}{*}{$\begin{array}{c}\text { Real } \\
\text { Distribution }\end{array}$} & Parameters & \multicolumn{3}{c}{ Classical Test } & \multicolumn{3}{c}{ Test with the BT } \\
\cline { 3 - 8 } LogNormal & $\mu=1, \sigma^{2}=0.25$ & 14.22 & 31.41 & 43.44 & 14.09 & 31.28 & 43.15 \\
LogNormal & $\mu=1, \sigma^{2}=1$ & 66.84 & 83.64 & 89.61 & 63.46 & 81.46 & 88.09 \\
BurrXII & $\eta=4, \tau=8, \lambda=1$ & 14.59 & 28.65 & 38.66 & 15.57 & 29.83 & 40.13 \\
BurrXII & $\eta=1, \tau=1, \lambda=2$ & 63.73 & 78.29 & 84.17 & 58.14 & 74.91 & 81.56 \\
Pareto & $\alpha=8$ & 3.91 & 11.31 & 18.46 & 3.45 & 10.68 & 17.82 \\
Pareto & $\alpha=2$ & 63.73 & 78.29 & 84.17 & 58.14 & 74.91 & 81.56 \\
GPD & $\sigma=1, \gamma=-1 / 16$ & 1.14 & 5.27 & 10.56 & 1.12 & 5.3 & 10.33 \\
GPD & $\sigma=1, \gamma=-1 / 4$ & 7.7 & 22.29 & 33.52 & 6.68 & 20.13 & 31.11 \\
Gamma & $\lambda=1 . \alpha=0.5$ & 1.04 & 4.54 & 9.33 & 0.92 & 4.34 & 9.71 \\
Gamma & $\lambda=1 . \alpha=1$ & 0.98 & 4.4 & 9.64 & 0.96 & 4.47 & 9.73 \\
Gamma & $\lambda=1 . \alpha=2.5$ & 0.93 & 4.44 & 9.8 & 0.99 & 4.58 & 9.85 \\
Gamma & $\lambda=1 . \alpha=5$ & 0.94 & 4.48 & 9.8 & 1.01 & 4.63 & 9.84 \\
Gamma & $\lambda=1 . \alpha=10$ & 0.95 & 4.52 & 9.79 & 1 & 4.61 & 9.81 \\
Control & $\mu=0, \sigma=0$ & - & - & - & 1.01 & 4.6 & 9.84 \\
\hline
\end{tabular}


Biased transformation and Goodness-of-Fit Test

Table 5: Percentage of rejections of $\mathrm{H}_{0}$ of the goodness-of-fit test for the assumed beta distributions for different levels of significance [\%] for the sample size $n=10$ and 25 (*distribution is truncated, scaled and/or moved)

\begin{tabular}{cccccccc} 
Real Distribution & \multirow{2}{*}{ Parameter } & \multicolumn{3}{c}{ BT and Anderson-Darling } & \multicolumn{3}{c}{ BT and Anderson-Darling } \\
& & \multicolumn{2}{c}{ Test for Normality for $\mathrm{n}=10$} & \multicolumn{2}{c}{ Test for Normality for $\mathrm{n}=25$} \\
& & $1 \%$ & $5 \%$ & $10 \%$ & $1 \%$ & $5 \%$ & $10 \%$ \\
\hline Beta & $\alpha=0.5, \beta=0.5$ & 1.00 & 5.33 & 10.76 & 0.82 & 4.43 & 9.31 \\
Beta & $\alpha=1, \beta=1$ & 1.04 & 5.51 & 10.73 & 0.86 & 4.50 & 9.38 \\
Beta & $\alpha=2, \beta=2$ & 1.04 & 5.57 & 10.64 & 0.83 & 4.53 & 9.52 \\
Beta & $\alpha=4, \beta=4$ & 1.09 & 5.54 & 10.64 & 0.85 & 4.58 & 9.49 \\
Beta & $\alpha=1, \beta=4$ & 1.05 & 5.46 & 10.77 & 0.88 & 4.53 & 9.42 \\
Beta & $\alpha=2, \beta=4$ & 1.06 & 5.53 & 10.65 & 0.85 & 4.56 & 9.53 \\
Beta & $\alpha=0.5, \beta=1$ & 0.99 & 5.35 & 10.65 & 0.85 & 4.48 & 9.40 \\
Beta & $\alpha=0.5, \beta=2$ & 1.01 & 5.31 & 10.62 & 0.83 & 4.54 & 9.32 \\
Beta & $\alpha=0.5, \beta=3$ & 1.02 & 5.28 & 10.64 & 0.86 & 4.55 & 9.37 \\
LogNormal $^{*}$ & $\mu=1, \sigma^{2}=0.25$ & 5.70 & 14.70 & 22.33 & 16.88 & 31.52 & 41.57 \\
LogNormal $^{*}$ & $\mu=1, \sigma^{2}=1.0$ & 6.72 & 16.80 & 25.30 & 22.34 & 39.47 & 49.90 \\
Gamma $^{*}$ & $\gamma=3, \lambda=1$ & 3.29 & 9.75 & 16.62 & 7.35 & 17.19 & 25.13 \\
Gamma $^{*}$ & $\gamma=1, \lambda=1$ & 2.31 & 8.10 & 14.45 & 4.26 & 12.35 & 19.24 \\
Normal $^{*}$ & $\mu=0, \sigma^{2}=1$ & 2.49 & 9.36 & 15.18 & 3.99 & 12.24 & 19.62 \\
Control $^{*}$ & $\mu=0, \sigma^{2}=1$ & 1.12 & 5.56 & 10.70 & 0.84 & 4.58 & 9.42 \\
\hline
\end{tabular}

Table 6: Percentage of rejections of $\mathrm{H}_{0}$ of the goodness-of-fit tests for the assumed beta distributions for different levels of significance [\%] for the sample size $\mathrm{n}=50$. (*distribution is truncated, scaled and/or moved)

\begin{tabular}{|c|c|c|c|c|c|c|c|c|c|c|}
\hline \multirow[t]{2}{*}{$\begin{array}{c}\text { Real } \\
\text { Distribution }\end{array}$} & \multirow[t]{2}{*}{ Parameters } & \multicolumn{3}{|c|}{$\chi^{2}$ Test } & \multicolumn{3}{|c|}{ Kolmogorov-Smirnov Test } & \multicolumn{3}{|c|}{$\begin{array}{c}\text { BT and Anderson-Darling } \\
\text { Test }\end{array}$} \\
\hline & & $1 \%$ & $5 \%$ & $10 \%$ & $1 \%$ & $5 \%$ & $10 \%$ & $1 \%$ & $5 \%$ & $10 \%$ \\
\hline Beta & $\alpha=0.5, \beta=0.5$ & 2.05 & 7.71 & 14.76 & 0 & 0.06 & 0.31 & 0.86 & 4.34 & 9.3 \\
\hline Beta & $\alpha=1, \beta=1$ & 2.13 & 7.99 & 15.08 & 0 & 0.05 & 0.2 & 0.88 & 4.49 & 9.5 \\
\hline Beta & $\alpha=2, \beta=2$ & 2.12 & 8.19 & 15.52 & 0 & 0.03 & 0.15 & 0.82 & 4.56 & 9.52 \\
\hline Beta & $\alpha=4, \beta=4$ & 2.15 & 8.19 & 15.12 & 0 & 0.01 & 0.07 & 0.84 & 4.56 & 9.53 \\
\hline Beta & $\alpha=1, \beta=4$ & 2.21 & 8.36 & 15.81 & 0 & 0.03 & 0.18 & 0.88 & 4.54 & 9.59 \\
\hline Beta & $\alpha=2, \beta=4$ & 2.25 & 8.5 & 16.21 & 0 & 0.02 & 0.1 & 0.84 & 4.54 & 9.57 \\
\hline Beta & $\alpha=0.5, \beta=1$ & 2.14 & 8.06 & 15.06 & 0 & 0.09 & 0.27 & 0.85 & 4.36 & 9.28 \\
\hline Beta & $\alpha=0.5, \beta=2$ & 2.2 & 8.16 & 15.33 & 0 & 0.1 & 0.37 & 0.84 & 4.29 & 9.38 \\
\hline Beta & $\alpha=0.5, \beta=3$ & 2.13 & 8.21 & 15.22 & 0 & 0.08 & 0.27 & 0.84 & 4.28 & 9.35 \\
\hline LogNormal ${ }^{*}$ & $\mu=1, \sigma^{2}=0.25$ & 12.54 & 28.68 & 40.75 & 0.63 & 4.93 & 11.28 & 37.06 & 55.86 & 65.86 \\
\hline LogNormal ${ }^{*}$ & $\mu=1, \sigma^{2}=1.0$ & 19.08 & 37.61 & 49.53 & 2.1 & 11.46 & 21.74 & 49.25 & 68.12 & 76.32 \\
\hline Gamma $^{*}$ & $\gamma=3, \lambda=1$ & 6.18 & 17.15 & 27.35 & 0.06 & 1.28 & 3.81 & 14.16 & 29.11 & 39.11 \\
\hline Gamma ${ }^{*}$ & $\gamma=1, \lambda=1$ & 4.83 & 14.22 & 23.38 & 0.04 & 0.8 & 2.32 & 7.55 & 18.92 & 27.99 \\
\hline Normal* & $\mu=0, \sigma^{2}=1$ & 5.02 & 14.96 & 24.18 & 0 & 0.24 & 1.03 & 7.04 & 18.07 & 26.67 \\
\hline Control & $\mu=0, \sigma^{2}=1$ & - & - & - & - & - & - & 0.84 & 4.61 & 9.54 \\
\hline
\end{tabular}


1

2

3

Biased transformation and Goodness-of-Fit Test

Table 7: Percentage of rejections of $\mathrm{H}_{0}$ of the goodness-of-fit tests for the assumed beta distributions for different levels of significance [\%] for the sample size $n=100$. (*distribution is truncated, scaled and/or moved.)

\begin{tabular}{|c|c|c|c|c|c|c|c|c|c|c|}
\hline \multirow[t]{2}{*}{ Real Distribution } & \multirow[t]{2}{*}{ Parameters } & \multicolumn{3}{|c|}{$\chi^{2}$ Test } & \multicolumn{3}{|c|}{ Kolmogorov-Smirnov Test } & \multicolumn{3}{|c|}{$\begin{array}{c}\text { BT and Anderson-Darling } \\
\text { Test }\end{array}$} \\
\hline & & $1 \%$ & $5 \%$ & $10 \%$ & $1 \%$ & $5 \%$ & $10 \%$ & $1 \%$ & $5 \%$ & $10 \%$ \\
\hline Beta & $\alpha=0.5, \beta=0.5$ & 2.24 & 7.46 & 13.32 & 0 & 0.08 & 0.49 & 1.27 & 5.1 & 10.11 \\
\hline Beta & $\alpha=1, \beta=1$ & 2.29 & 7.48 & 13.23 & 0 & 0.04 & 0.35 & 1.27 & 5.12 & 10.3 \\
\hline Beta & $\alpha=2, \beta=2$ & 2.37 & 7.72 & 13.7 & 0 & 0.03 & 0.16 & 1.29 & 5.22 & 10.3 \\
\hline Beta & $\alpha=4, \beta=4$ & 2.55 & 7.73 & 13.6 & 0 & 0.01 & 0.1 & 1.29 & 5.12 & 10.29 \\
\hline Beta & $\alpha=1, \beta=4$ & 2.31 & 7.77 & 13.76 & 0 & 0.05 & 0.25 & 1.28 & 5.1 & 10.21 \\
\hline Beta & $\alpha=2, \beta=4$ & 2.57 & 8.18 & 14.31 & 0 & 0.01 & 0.13 & 1.31 & 5.16 & 10.25 \\
\hline Beta & $\alpha=0.5, \beta=1$ & 2.24 & 7.47 & 13.31 & 0 & 0.1 & 0.56 & 1.23 & 5.06 & 9.99 \\
\hline Beta & $\alpha=0.5, \beta=2$ & 2.32 & 7.46 & 13.43 & 0 & 0.08 & 0.46 & 1.25 & 5.03 & 9.98 \\
\hline Beta & $\alpha=0.5, \beta=3$ & 2.32 & 7.6 & 13.29 & 0 & 0.07 & 0.41 & 1.22 & 5.06 & 9.93 \\
\hline LogNormal $^{*}$ & $\mu=1, \sigma^{2}=0.25$ & 25.19 & 45.77 & 58.76 & 4.26 & 16.05 & 27.64 & 69.88 & 84.72 & 89.94 \\
\hline LogNormal $^{*}$ & $\mu=1, \sigma^{2}=1.0$ & 39.17 & 61.08 & 71.75 & 11.27 & 35.3 & 52.58 & 84.01 & 93.39 & 96.09 \\
\hline Gamma* & $\gamma=3, \lambda=1$ & 8.8 & 21.39 & 31.32 & 0.74 & 4.51 & 9.11 & 29.8 & 49.67 & 60.75 \\
\hline Gamma $^{*}$ & $\gamma=1, \lambda=1$ & 5.92 & 15.51 & 23.76 & 0.25 & 2.49 & 5.88 & 15.89 & 31.18 & 42.14 \\
\hline Normal* & $\mu=0, \sigma^{2}=1$ & 4.93 & 14.31 & 22.88 & 0.04 & 0.43 & 1.51 & 12.79 & 27.82 & 38.96 \\
\hline Control & $\mu=0, \sigma^{2}=1$ & - & - & - & - & - & - & 1.25 & 5.08 & 10.28 \\
\hline
\end{tabular}

